\title{
MEASUREMENTS OF PARTICLE VELOCITIES AND TRAJECTORIES IN A WAVE- CURRENT MOTION USING PARTICLE IMAGE VELOCIMETRY
}

\author{
Motohiko Umeyama ${ }^{1}$, Tetsuya Shintani ${ }^{1}$ and Shinya Watanabe ${ }^{1}$
}

\begin{abstract}
This article deals with some physical aspects of a water particle under surface waves, which propagate with or without a current in a constant water depth, using an imaging technique. The use of particle image velocimetry (PIV) made it possible to investigate the velocity and trajectory of each individual water particle. The velocity vector fields and its vertical distributions were estimated at several phases in one wave cycle. The theory of progressive waves based on finite-amplitude approximation was adapted to express the velocity potential, surface displacement and angular frequency. The PIV result showed suitable agreement with a solution solved to the third order by a perturbation method. In addition, the distributions of horizontal and vertical velocity components by the PIV measurement were compared with those by an EC meter. These attempts proved the ability of the PIV technique to accurately measure both temporal and spatial variations of the velocity. This technique was applied to the prediction of particle trajectory in a Eulerian scheme. In the method, the surrounding grid velocities were used to identify a Lagrangian velocity. The measured particle path was compared with the positions found theoretically by integrating the Eulerian velocity to the second order of a Taylor series expansion.
\end{abstract}

Keywords: Surface waves; Velocity; Trajectory; Flow measurement; Imaging techniques

\section{INTRODUCTION}

Airy (1845) developed a small-amplitude wave theory in which the surface profile and velocity motion is sinusoidal and the trajectory is circler or elliptical in water of uniform depth. Since then, a number of researches for the gravity waves have performed to obtain satisfactory mathematical solutions in the Eulerian system. Stokes (1847) presented a second-order theory for finite amplitude waves by introducing trigonometric series. The Stokes wave profile is much more peaked at the crest and flatter at the trough than the sinusoidal form. In addition, the second-order approximation results in the incomplete closure of the particle trajectory that becomes quite distorted due to nonlinearity. The particle motion for irrotational waves leads to a net mass-transport (called Stokes drift) in the direction of wave propagation. Major attention has been given to the problem of higher-order wave profile in the Eulerian system for the last century. For instance, the Stokes method has been extended to the third order by Borgman and Chappelear (1958), and to the fifth order by Skjelbreia and Hendrickson (1961) and Fenton (1985). The subject of the water particle velocity and trajectory may be of considerable importance, but the number of such investigations is limited. It is not convenient to describe the trajectory of particles and mass transport under a wave motion in the Lagrangian system. Thus the Eulerian approach is more frequently used in solving fluid dynamic problems. Recently, Constantin (2006) and Constantin \& Strauss (2009) solved a set of differential equations to investigate the particle paths in a fixed Eulerian system. Generally, to obtain the particle trajectory, the particle velocity from the Eulerian solutions has been expanded to the higher order and integrated about its mean position.

A series of photographs taken by Wallet \& Ruellan (1950) is the first set of the trajectory motion. For the latter half of $20^{\text {th }}$ century the use of hot-wire velocimetry, EC meters, or laser Doppler anemometers (LDA) has measured several types of flow velocities. However, these point-measurement techniques have limited spatial resolution, while providing high temporal resolution. Recently, visualization techniques have played a more important role, as these yield both qualitative and quantitative insights in fluid flows. Significant developments in particle image velocimetry (PIV) and particle tracking velocimetry (PTV) have enabled the visualization of velocity fields and water particle paths. These flow visualization techniques can be seen in Adrian (1991), Willert \& Gharib (1991), and Grue et al. (2004). From a Eulerian prospective, the motion of an incompressible fluid is distinctive if the velocity vectors can occupy an instantaneous velocity field. As PIV is used to represent a regular array of velocity vectors, it is convenient to define the Eulerian velocity based upon the average particle motion in the possible space. In contrast, we generally use PTV for a Lagrangian description of the motion in a system where it traces individual particle path from a sequence of images. Quantitative results for Lagrangian fluid motion can be obtained through computerized analyses of the seed-particle images in the modern PTV technique. In the Hydraulic laboratory of Tokyo Metropolitan University,

\footnotetext{
${ }^{1}$ Department of Civil \& Environmental Engineering, Tokyo Metropolitan University, 1-1 Minami-ohsawa, Hachioji, Tokyo 192-0397, Japan
} 
using a PIV system with an Nd:YAG laser and a CCD camera, Shimizu, Shintani \& Umeyama (2006) measured the velocity vector fields of internal waves over a uniform slope. Recently, Umeyama (2008) and Umeyama \& Shinomiya (2009) set up an apparatus to measure the instantaneous velocity of internal waves propagating in a two-layer fluid system, attempting a PIV system with halogen lamp as a light source and some high-definition digital video cameras.

In this study, we focus our attention on surface waves propagating with or without a steady current. A third-order perturbation solution is derived so as to express the nonlinearity of water particle velocity. The particle displacement is also obtained by integrating the second-order horizontal and vertical velocities over time. To measure and analyze the water particle velocity and trajectory, we used a PIV system with two Nd:YAG lasers. We tested two different tracers such as DIAION and micro bubbles. Validation results are also presented from the comparison of measurements via the PIV system to those via the EC meter. An alternative measurement technique is proposed to describe the instantaneous velocity fields, horizontal and vertical velocity distributions and water particle trajectories of Stokes waves in the presence of a current.

\section{THEORIES}

\section{The governing equations}

The waves propagating on the water surface are approximated to be two-dimensional when the motion is identical in any direction parallel to the crest line. Now we consider a cross section of the wave field that is perpendicular to the crest line with Cartesian coordinates $(x, z)$, the $x$-axis being the direction of wave propagation and the $z$-axis pointing vertically upward, while the origin lies on the mean sea level. The wave motion is assumed to be irrotational in an inviscid and incompressible fluid. Let $u(x, z, t)$ and $w(x, z, t)$ be the horizontal and vertical components of velocity at a time, $t$. In terms of a velocity potential $\phi(x, z, t)$, the velocity components can be expressed as

$$
\begin{aligned}
& u(x, z, t)=\phi_{x} \\
& w(x, z, t)=\phi_{z}
\end{aligned}
$$

Homogeneity is a good approximation for water, and it implies the equation of mass conservation that satisfies the Laplace equation:

$$
\phi_{x x}+\phi_{z z}=0
$$

We now present the boundary conditions for the water wave problem. The dynamic boundary condition is

$$
g \eta+\phi_{t}+\frac{1}{2}\left(\phi_{x}^{2}+\phi_{z}^{2}\right)=0 \quad \text { on } z=\eta
$$

where $g=$ acceleration due to gravity. The kinematical boundary conditions are

$$
\eta_{t}+\phi_{x} \eta_{x}-\phi_{z}=0 \quad \text { on } z=\eta
$$

and

$$
\phi_{z}=0 \quad \text { on } z=-h
$$

expressing the fact that the same particles always form the free water surface and the flow is tangent to the horizontal bed.

\section{Nonlinear water waves with a uniform current}

In the finite amplitude wave theory, the perturbation technique that can yield uniformly valid expansions is chosen to solve Eqs.3, 4, 5 and 6. The wave motion is periodic in time and space. Thus the perturbation variables can be expressed by a series of which each term is a power of $n^{\text {th }}$ order in terms of the dimensionless perturbation parameter and has sinusoidal functions with an angular frequency.

$$
\begin{aligned}
& \phi=U x+\sum_{n=1}^{\infty} \varepsilon^{n} \phi^{(n)} \\
& \eta=\sum_{n=1}^{\infty} \varepsilon^{n} \eta^{(n)}
\end{aligned}
$$




$$
\sigma=\sum_{n=0}^{\infty} \varepsilon^{n} \sigma^{(n)}
$$

where $\varepsilon=$ perturbation parameter and $\sigma=$ angular frequency. The quantities of $\phi^{(n)}, \eta^{(n)}$ and $\sigma^{(n)}$ are by an order $O\left(\varepsilon^{n}\right)$.

Now we replace the free-surface boundary conditions in Eqs. 4 and 5 by the conditions to be satisfied for $z=0$ instead of $z=\eta$. The boundary conditions for the first, second, and third powers can be obtained by expanding Eqs. 4 and 5 into a Taylor series in $z$, and substituting Eqs.7, 8 and 9 into them and Eq.7 into Eq.6. Umeyama (2010) obtained the results of a third-order solution as follows: The first-order solution is

$$
\begin{aligned}
& \phi^{(1)}=\frac{1}{k^{2}} \sigma_{A} \frac{\cosh k(z+h)}{\sinh k h} \sin (k x-\sigma t) \\
& \eta^{(1)}=\frac{1}{k} \cos (k x-\sigma) \\
& \sigma^{(0)}=U k+\sigma_{A}
\end{aligned}
$$

in which $k=$ wave number and $\sigma_{A}=\sqrt{g k \tanh k h}$.

The second-order solution is

$$
\begin{aligned}
& \phi^{(2)}=\frac{1}{k^{2}} \sigma_{A} \alpha_{2} \cosh 2 k(z+h) \sin 2(k x-\sigma) \\
& \eta^{(2)}=\frac{1}{k} \beta_{22} \cos 2(k x-\sigma t) \\
& \sigma^{(1)}=0
\end{aligned}
$$

in which

$$
\begin{aligned}
& \alpha_{2}=\frac{3 g k\left(1-\tanh ^{2} k h\right)}{2\left(1+\tanh ^{2} k h\right)\left\{2\left(\sigma^{(0)^{2}}+U^{2} k^{2}\right)-k\left(g \tanh 2 k h+\sigma^{(0)} U\right)\right\}} \\
& \beta_{22}=\frac{1}{2}\left\{\frac{3 g k\left(1-\tanh ^{2} k_{I} h\right)}{2\left(\sigma^{(0)^{2}}+U^{2} k^{2}\right)-k\left(g \tanh 2 k h+\sigma^{(0)} U\right)}+\frac{1+\tanh ^{2} k h}{2 \tanh k h}\right\}
\end{aligned}
$$

The third-order solution is

$$
\begin{aligned}
\phi^{(3)} & =\frac{1}{k^{2}} \sigma_{A} \alpha_{3} \cosh 3 k(z+h) \sin 3(k x-\sigma) \\
\eta^{(3)} & =\frac{1}{k}\left\{\beta_{31} \cos (k x-\sigma)+\beta_{33} \cos 3(k x-\sigma)\right\} \\
\sigma^{(2)} & =\sigma_{A}\left(\beta_{22} \frac{1-\tanh ^{2} k h}{4 \tanh k h}-\alpha_{2} \frac{\tanh k h \tanh 2 k h-2}{2 \tanh 2 k h}+\frac{3 U k}{16 \sigma_{A}}+\frac{11}{16}\right)
\end{aligned}
$$

in which

$$
\begin{aligned}
& \alpha_{3}=\frac{g k \tanh 3 k h}{3 g k \tanh 3 k-9\left(\sigma^{(0)^{2}}+U^{2} k^{2}\right)+18 \sigma^{(0)} U k}\left\{\frac{3 \beta_{22}\left(\tanh ^{2} k h-1\right)}{2 \tanh k h}+\frac{3 \alpha_{2}\left(3 \tanh ^{2} k h-2\right)}{\tanh 2 k h}-\frac{3 U k}{8 \sigma_{A}}-\frac{3}{8}\right\} \\
& \beta_{31}=\beta_{22} \frac{\tanh ^{2} k h+1}{4 \tanh k h}+\frac{\alpha_{2}}{2} \tanh k h-\frac{13 U k}{16 \sigma_{A}}-\frac{5}{16} \\
& \beta_{33}=\frac{\beta_{22}}{2} \tanh k h-\frac{U k}{8 \sigma_{A}}+\frac{3 \alpha_{2} \tanh k h \tanh 2 k h-1}{\tanh 2 k h}+\frac{3 \alpha_{3}\left(\sigma^{(0)}+U k\right) \sigma_{A}}{g k \tanh 3 k h}
\end{aligned}
$$

\section{Particle trajectories}

If $(x(t), z(t))$ is the instantaneous water particle position below the linear wave at a time $t$, then, the horizontal and vertical velocities become 


$$
\frac{d x}{d t}=u=\frac{\partial \phi}{\partial x} \text { and } \frac{d z}{d t}=w=\frac{\partial \phi}{\partial z}
$$

When the mean position of a water particle is given at $(\bar{x}, \bar{z})$, the path of the particle is denoted as

$$
x(t)=\bar{x}+\varsigma(t) \text { and } z(t)=\bar{z}+\xi(t)
$$

where $\varsigma=$ horizontal displacement and $\xi=$ vertical displacement.

Expansion of Eq.19 in Taylor series yields

$$
\begin{aligned}
& \frac{d \varsigma}{d t}=\frac{\partial \phi(\bar{x}+\varsigma, \bar{z}+\xi)}{\partial x}=\left(\frac{\partial \phi}{\partial x}\right)_{\bar{x}, \bar{z}}+\varsigma\left(\frac{\partial^{2} \phi}{\partial x^{2}}\right)_{\bar{x}, \bar{z}}+\xi\left(\frac{\partial^{2} \phi}{\partial x \partial z}\right)_{\bar{x}, \bar{z}}+\cdots \\
& \frac{d \xi}{d t}=\frac{\partial \phi(\bar{x}+\varsigma, \bar{z}+\xi)}{\partial z}=\left(\frac{\partial \phi}{\partial z}\right)_{\bar{x}, \bar{z}}+\varsigma\left(\frac{\partial^{2} \phi}{\partial x \partial z}\right)_{\bar{x}, \bar{z}}+\xi\left(\frac{\partial^{2} \phi}{\partial z^{2}}\right)_{\bar{x}, \bar{z}}+\cdots
\end{aligned}
$$

For first approximation, we assume that $\varsigma$ and $\xi$ be small quantities and therefore $x(t)=\bar{x}+\varsigma(t)$ and $z(t)=\bar{z}+\xi(t)$ can be replaced with $x(t)=\bar{x}$ and $z(t)=\bar{z}$. Integrating Eqs.21 and 22 to the first term with respect to $t$ yields

$$
\begin{aligned}
\varsigma=U t & -\varepsilon \frac{\sigma_{A}}{k \sigma} \frac{\cosh k(\bar{z}+h)}{\sinh k h} \sin (k \bar{x}-\sigma)-\varepsilon^{2} \frac{\sigma_{A}}{k \sigma} \alpha_{2} \frac{\cosh 2 k(\bar{z}+h)}{\sinh 2 k h} \sin 2(k \bar{x}-\sigma) \\
& +\varepsilon^{3} \frac{\sigma_{A}}{k \sigma} \alpha_{3} \frac{\cosh 3 k(\bar{z}+h)}{\sinh 3 k h} \sin 3(k x-\sigma)+\cdots \\
\xi= & \varepsilon \frac{\sigma_{A}}{k \sigma} \frac{\sinh k(\bar{z}+h)}{\sinh k h} \cos (k \bar{x}-\sigma)+\varepsilon^{2} \frac{\sigma_{A}}{k \sigma} \alpha_{2} \frac{\sin 2 k(\bar{z}+h)}{\sinh 2 k h} \cos 2(k \bar{x}-\sigma t) \\
+ & \varepsilon^{3} \frac{\sigma_{A}}{k \sigma} \alpha_{3} \frac{\sinh 3 k(\bar{z}+h)}{\sinh 3 k h} \cos 3(k \bar{x}-\sigma)+\cdots
\end{aligned}
$$

In order to obtain the second approximation, we substitute Eqs.23 and 24 into Eqs.21 and 22. So that the solution for the instantaneous water particle position becomes

$$
\begin{aligned}
x(t)=\bar{x}+U t & +\varepsilon \frac{\sigma_{A}}{\sigma^{2}}\left(\frac{\sigma}{k}-U\right) \frac{\cosh k(\bar{z}+h)}{\sinh k h} \sin (k \bar{x}-\sigma t) \\
& -\varepsilon^{2}\left[\frac{\sigma_{A}}{\sigma^{2}} \alpha_{2}\left(\frac{\sigma}{4 k}-U\right) \frac{\cosh 2 k(\bar{z}+h)}{\sinh 2 k h} \sin 2(k \bar{x}-\sigma t)\right. \\
& \left.-\frac{\sigma_{A}^{2}}{4 k \sigma^{2} \sinh ^{2} k h}\{2 \sigma t \cosh 2 k(\bar{z}+h)+\sin 2(k \bar{x}-\sigma t)\}\right] \\
& +\varepsilon^{3}\left[\frac{\sigma_{A}}{\sigma^{2}} \alpha_{3}\left(\frac{\sigma}{k}-U\right) \frac{\sinh 3 k(\bar{z}+h)}{\sinh 3 k h} \sin 3(k \bar{x}-\sigma t)\right. \\
z(t)=\bar{z}+ & \left.\frac{\sigma_{A}}{\sigma^{2}}\left(\frac{\sigma}{k}+U\right) \frac{5 \sigma_{A}^{2}}{6 k \sigma^{2}} \alpha_{2}\{3 \cosh 3 k(\bar{z}+h) \sin (k \bar{x}-\sigma t)-\cosh k(\bar{z}+h) \sin 3(k \bar{x}-\sigma t)\}\right] \\
+ & \varepsilon^{2} \frac{\sigma_{A}}{\sigma^{2}} \alpha_{2}\left(\frac{\sigma}{k}+U\right) \frac{\sinh 2 k(\bar{z}+h)}{\sinh 2 k h} \cos (k \bar{x}-\sigma t) \\
+ & \varepsilon^{3}\left[\frac{\sigma_{A}}{\sigma^{2}} \alpha_{3}\left(\frac{\sigma}{k}+U\right) \frac{\sinh 3 k(\bar{z}+h)}{\sinh 3 k h} \cos 3(k \bar{x}-\sigma)\right. \\
+ & \left.\frac{\sigma_{A}^{2}}{2 k \sigma^{2}} \alpha_{2}\{3 \sinh 3 k(\bar{z}+h) \cos (k \bar{x}-\sigma t)-\sinh k(\bar{z}+h) \cos 3(k \bar{x}-\sigma t)\}\right]
\end{aligned}
$$




\section{EXPERIMENTS}

Experiments were carried out in a wave tank $25.0 \mathrm{~m}$ long, $0.7 \mathrm{~m}$ wide, and $1.0 \mathrm{~m}$ deep (see Figure 1). A piston-type wave generator was placed at one end of the tank. The water was recirculated via an $8 \mathrm{~cm}$-diameter pipe. The test section was located in the area from $14.0 \mathrm{~m}$ to $15.0 \mathrm{~m}$ downstream of the wave paddle. The water depth was $30 \mathrm{~cm}$ during all tests and the wave period was $1.0 \mathrm{~s}$ for several different wave-paddle motions. A summary of the experimental conditions is presented in Table 1. Runs W1-W4 are for regular waves without an artificially generated current. Runs WCF1-WCF4 are the waves of Runs W1-W4 superimposed over a forward current whose depth-averaged velocity is approximately $8.0 \mathrm{~cm} / \mathrm{s}$.

For measurement of water particle velocities, we have employed PIV based on a singleexposure image. The PIV technique evaluates the instantaneous velocities through recording the position of images of small tracers that are suspended in the fluid at successive instants in time. In practice, when two successive images of tracers illuminated in a thin and intense light sheet are acquired, the velocity can be calculated from the known time difference and measured displacement. As PIV seeks the mean displacement of a small group of tracers, it may be suited to a study of wave motion in a Eulerian system. The representation of the velocity vector field is a typical example of PIV. In addition to such popular use, we apply the PIV result to a particle tracking process.

The instantaneous water particle velocity was measured in a $92.0-\mathrm{cm}-$ long and $36.0-\mathrm{cm}$-high area (one glass panel). A new PIV system consisted of two frequency-doubled dual Nd:YAG lasers having a 50-mW energy. A 2-mm light sheet was emitted from the upper side: this light sheet had very uniform intensity and was well suited to PIV measurement over large areas. The system used two highdefinition digital video cameras with a maximum resolution of $2016 \times 1134$ pixels. These video cameras were arranged in a line $2 \mathrm{~m}$ from the sidewall of the wave tank. Each video camera had a different field of view and covered an area up to $64.0 \mathrm{~cm} \times 36.0 \mathrm{~cm}$ (frame rate 16:9). The field of view of the video cameras overlapped by $15 \%$ in the glass panel. The water in the tank was seeded with DIAION (DK-FINE HP20), having a grain size of $0.25 \mathrm{~mm}$ and specific gravity of 1.01 . The interrogation window was $21 \times 21$ pixels, while the candidate region was $42 \times 42$ pixels. Vector fields were obtained with the PIV system by processing a pair of image frames. The PIV algorithm was based on the cross-correlation method. The resultant displacement of topographic features for two image frames spaced at $\Delta t=0.1 \mathrm{~s}$ was chosen for direct calculation of the velocity vectors. After the PIV

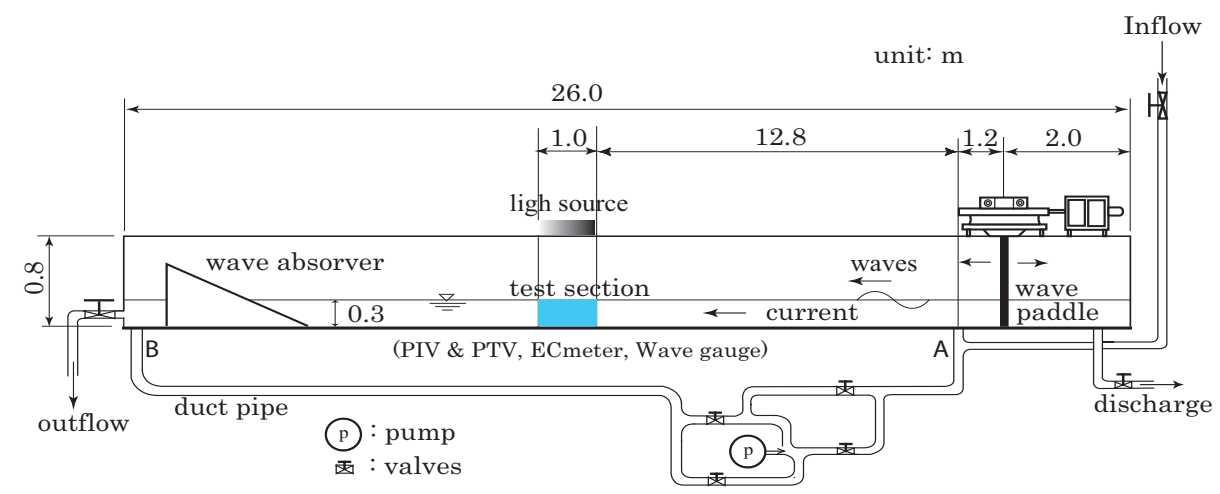

Figure 1 Sketch of apparatus.

Table 1. Experimental conditions.
\begin{tabular}{|c|c|c|c|c|c|c|}
\hline & W1 & W2 & W3 & WC1 & WC2 & WC3 \\
\hline Depth-averaged velocity $(\mathrm{cm} / \mathrm{s})$ & 0.0 & 0.0 & 0.0 & -8.0 & -8.0 & -8.0 \\
\hline Wave height $(\mathrm{cm})$ & 1.0 & 2.1 & 3.1 & 1.0 & 2.0 & 3.1 \\
\hline Wave period (s) & 1.0 & 1.0 & 1.0 & 1.0 & 1.0 & 1.0 \\
\hline
\end{tabular}



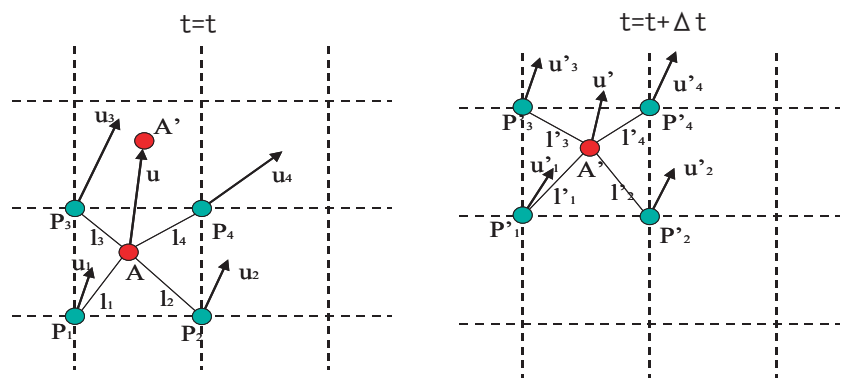

Figure 2 Lagrangian particle trajectory on Eulerian grids.

measurement gave the velocity available at spatially discrete nodal locations in a Eulerian scheme, we estimated the particle velocity and location. Fig. 2 depicts the motion of a particle within a tracking time step $\Delta t$ along an arbitrary trajectory of a particle across a general mesh of quadrilateral cells. The algorithm has two steps: (1) the velocity value of a Lagrangian point (A) at time $t$ is obtained by interpolating the neighboring velocity values $\left(\mathrm{u}_{1}\right.$ at $\mathrm{P}_{1}, \mathrm{u}_{2}$ at $\mathrm{P}_{2}, \mathrm{u}_{3}$ at $\mathrm{P}_{3}$, and $\mathrm{u}_{4}$ at $\left.\mathrm{P}_{4}\right)$; and (2) the particle associated with the Lagrangian point at $t$ is traced to a hypothetical location (A') at $t+\Delta t$. Thus these Lagrangian velocities are

$$
u=\frac{\frac{u_{1}}{l_{1}}+\frac{u_{2}}{l_{2}}+\frac{u_{3}}{l_{3}}+\frac{u_{4}}{l_{4}}}{\frac{1}{l_{1}}+\frac{1}{l_{2}}+\frac{1}{l_{3}}+\frac{1}{l_{4}}} \text { at } t=t \text {, and } \quad u^{\prime}=\frac{\frac{u_{1}^{\prime}}{l_{1}^{\prime}}+\frac{u_{2}^{\prime}}{l_{2}^{\prime}}+\frac{u_{3}^{\prime}}{l_{3}^{\prime}}+\frac{u_{4}^{\prime}}{l_{4}^{\prime}}}{\frac{1}{l_{1}^{\prime}}+\frac{1}{l_{2}^{\prime}}+\frac{1}{l_{3}^{\prime}}+\frac{1}{l_{4}^{\prime}}} \text { at } t=t+\Delta t
$$

In addition to DIAION for the tracer particle, we used micro bubbles of which the typical diameter is approximately 50 microns in water. These bubbles can remain suspended in the water for relatively longer period, as they contain oxygen or air. The gas within them dissolves gradually into the water and finally they disappear. Four micro bubble generators were set along a centerline on the bottom of the wave tank. Each generator was $0.5 \mathrm{~cm}$ diameter and $10.0 \mathrm{~cm}$ long, and its face was covered with a polymer film to control the formation of Nano-scale porous structures. The thickness of the film was 20 to $60 \mu \mathrm{m}$, the distance between each Nano-porous structure was 20 to $30 \mu \mathrm{m}$, and the width was 4 to $10 \mu \mathrm{m}$. After an image was captured, it was analyzed by applying the threshold to remove the noise. Then a cross-correlation method was performed to calculate the particle displacement. The interrogation area for velocity calculation was $64 \times 64$ pixels.

\section{RESULTS}

Figure 3 shows instantaneous velocity vector maps at an interval of $0.25 \mathrm{~s}$ for Runs W1 and W3 in a PIV measurement. Waves propagate from right to left. As the water particle under progressive waves stampedes from the area nearby a node just behind the crest and gathers around another node to maintain a surface profile, one can estimate the surface displacement from the velocity vector map at each phase. The extreme value of the horizontal velocity appears at the crest or trough positions where the water surface is maximum or minimum. An array of clockwise and counterclockwise circulations exists in the wave tank and it moves in the wave direction. Each pair of counter-rotating circulations seems to be nearly symmetric for Run W1. With increasing wave height, the experimental result provides that the velocities increase exponentially with elevation above the bottom. Although we cannot judge whether a particle beneath a crest moves faster in the forward direction than beneath a trough in the backward direction, it is clear that the nonlinear effect appears with increasing wave height. A pair of counter-rotating vortices becomes asymmetric for Run W3. Figure 4 depicts the corresponding velocity fields for waves following a steady current (Runs WC1 and WC3). The flow is no longer symmetric with respect to a vertical line drawn beneath the nodal point. There is no vortex that maintains a quasi-elliptic form. For the shorter wave case, current velocities are somewhat affected by the waves. As the wave height increases, the increase in the flow velocity becomes more evident, particularly beneath the near-crest layer. The flow area of the faster-moving fluids along the wave tank becomes wider. It is also evident that the horizontal velocity decreases and the backward-flow area appears when the trough of a larger wave approaches. 

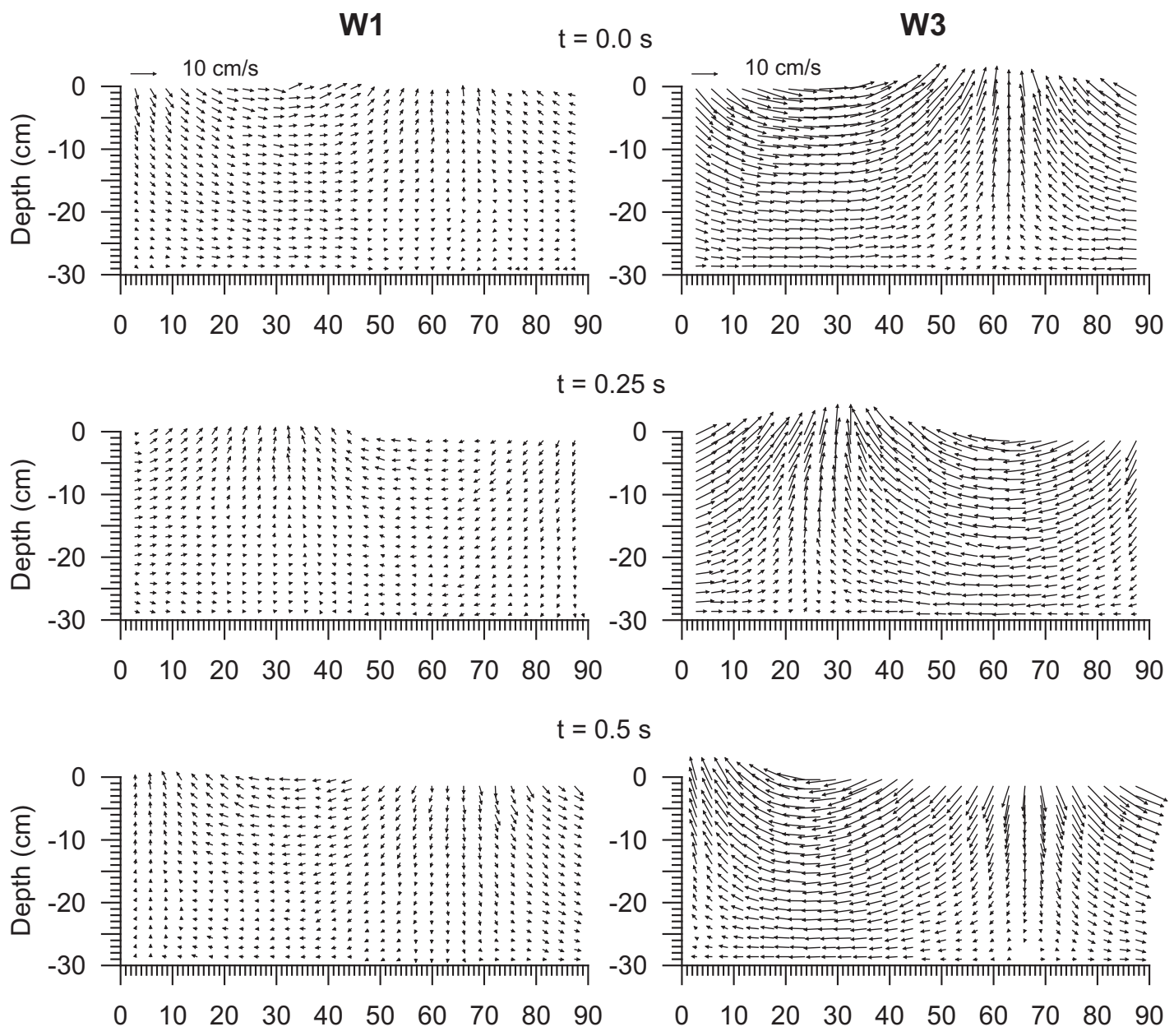
$t=0.75 s$
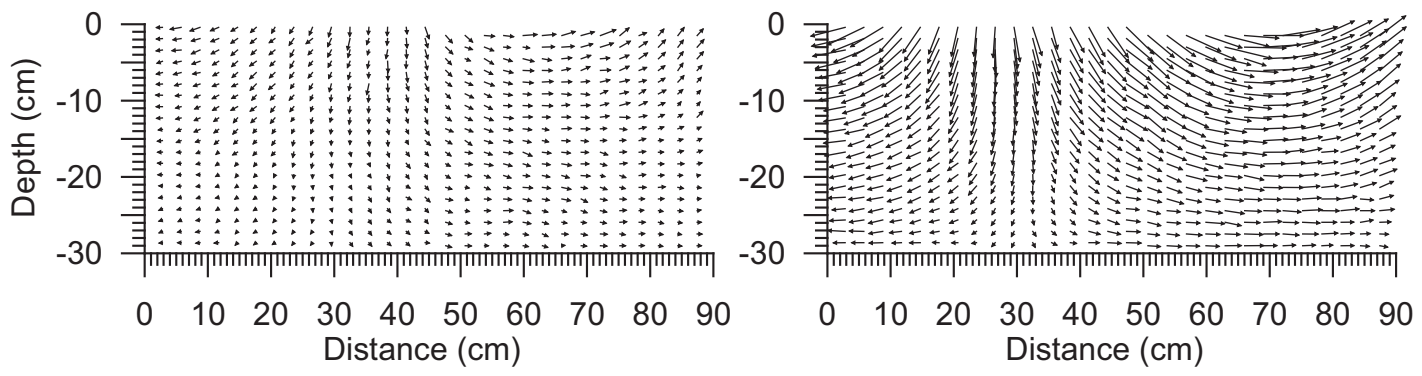

Figure 3 Instantaneous velocity fields by PIV measurement for wave-alone cases. 

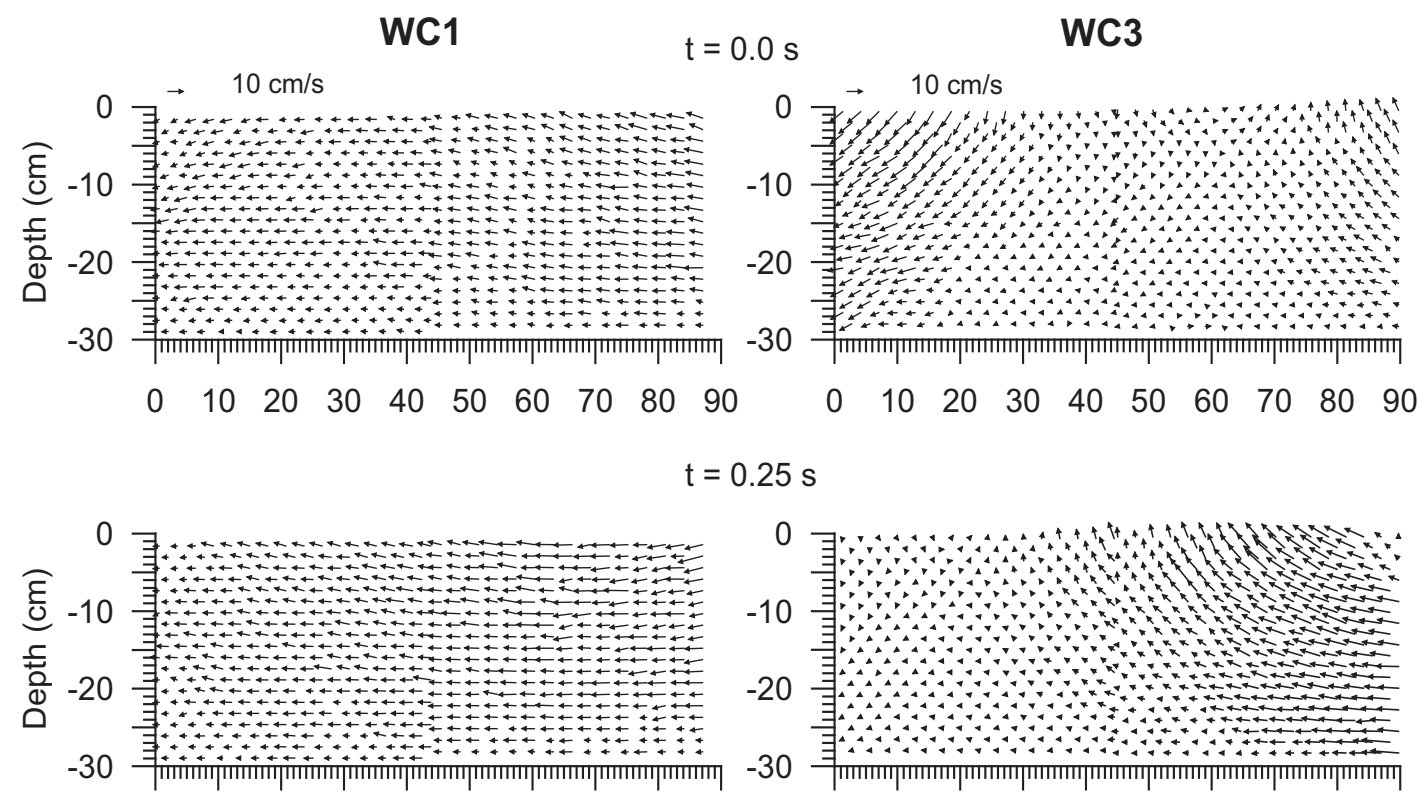

$\begin{array}{llllllllllllllllllll}0 & 10 & 20 & 30 & 40 & 50 & 60 & 70 & 80 & 90 & 0 & 10 & 20 & 30 & 40 & 50 & 60 & 70 & 80 & 90\end{array}$

$$
t=0.5 s
$$
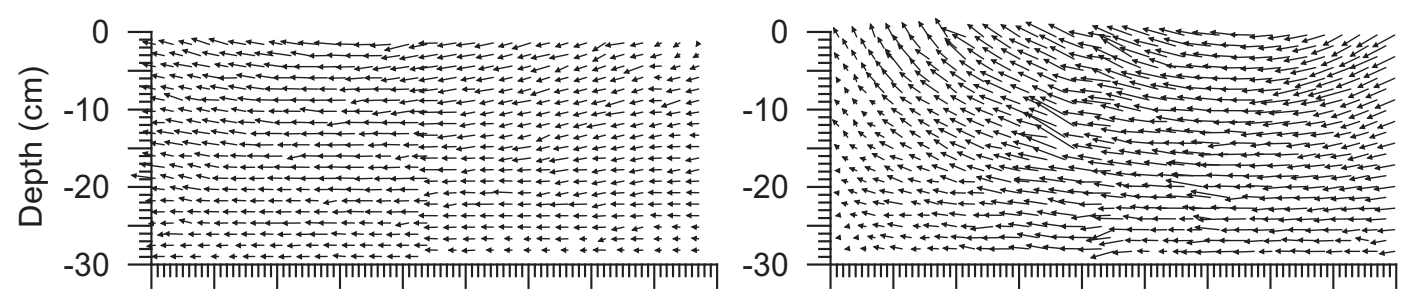

$\begin{array}{llllllllll}0 & 10 & 20 & 30 & 40 & 50 & 60 & 70 & 80 & 90\end{array}$

$\begin{array}{llllllllll}0 & 10 & 20 & 30 & 40 & 50 & 60 & 70 & 80 & 90\end{array}$ $t=0.75 s$
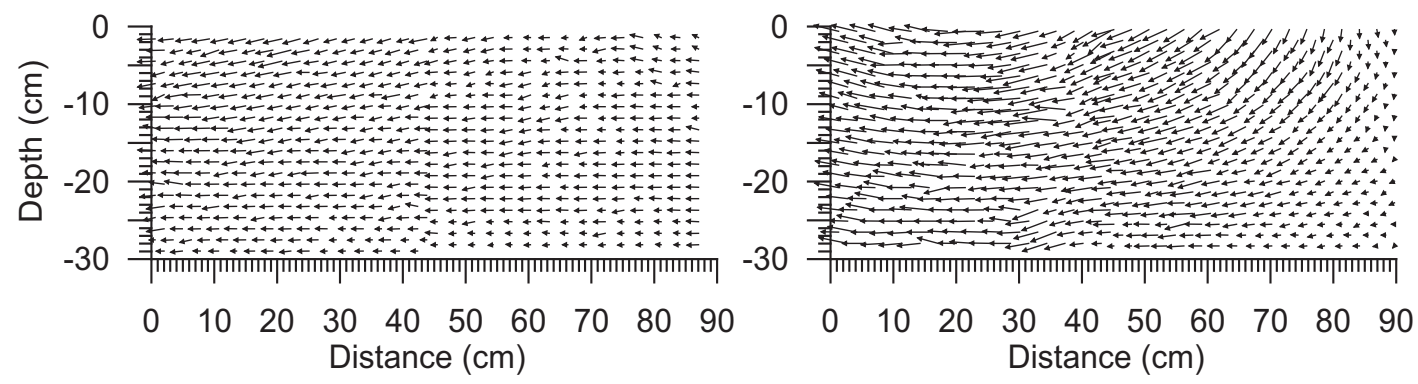

Figure 4 Instantaneous velocity fields by PIV measurement for wave-current cases. 
It is of great interest to compare the experimental velocity at a cross-section with the theoretical velocity. These velocity profiles at different phase values are shown in Figure 5. The calculations of the horizontal and vertical velocity components were carried out by the third-order Stokes approximation. As seen in the horizontal velocity profiles, the PIV result matches the theoretical prediction for most phases, except at the moment when the flow reverses. It is also noted that the experimental horizontal velocity gradually starts to evolve from the theoretical curve at the deeper depth with the increase of the wave height. The PIV plot for each experimental case shows significant differences in comparison to the corresponding EC plot at several elevations. The theoretical result of vertical velocity shows a good agreement with these PIV data in both experimental cases. The comparison of the nonintrusive (PIV)
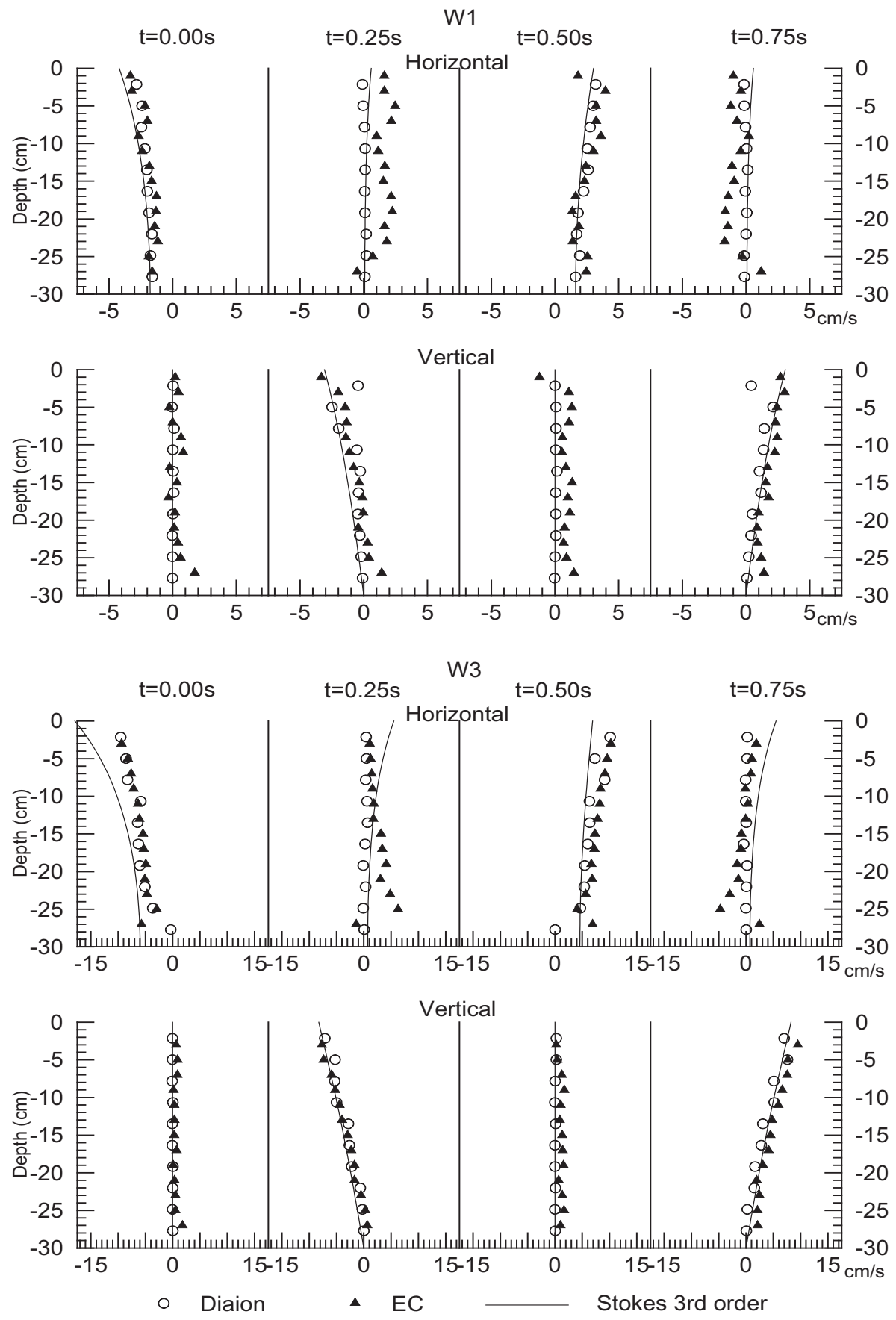

Figure 5 Horizontal and vertical velocity profiles for wave-alone cases. 
and intrusive (EC) measurements is most convincing in the region between -5 and $-25 \mathrm{~cm}$, aside from a scatter of data. Figure 6 depicts similar distributions of the horizontal and vertical velocity components for waves in a steady current. For each individual velocity component, comparisons are made between two profiles using DIAION and micro bubbles. The results obtained by DIAION and micro bubbles seem similar trends. Nevertheless, there is a difference for the vertical velocity component at the relatively higher elevations for Run WC1 when the wave crest or trough arrives. These differences could have some explanations. First, micro bubbles show a tendency to rise toward the water surface while DIAION sinks gradually in the still water because of its specific density of 1.01. Second, the PIV technique also requires the use of a large laser power. The laser of $50 \mathrm{~mW}$ is inadequate to detect smaller bubbles that are relatively stable in the wave-current motion.
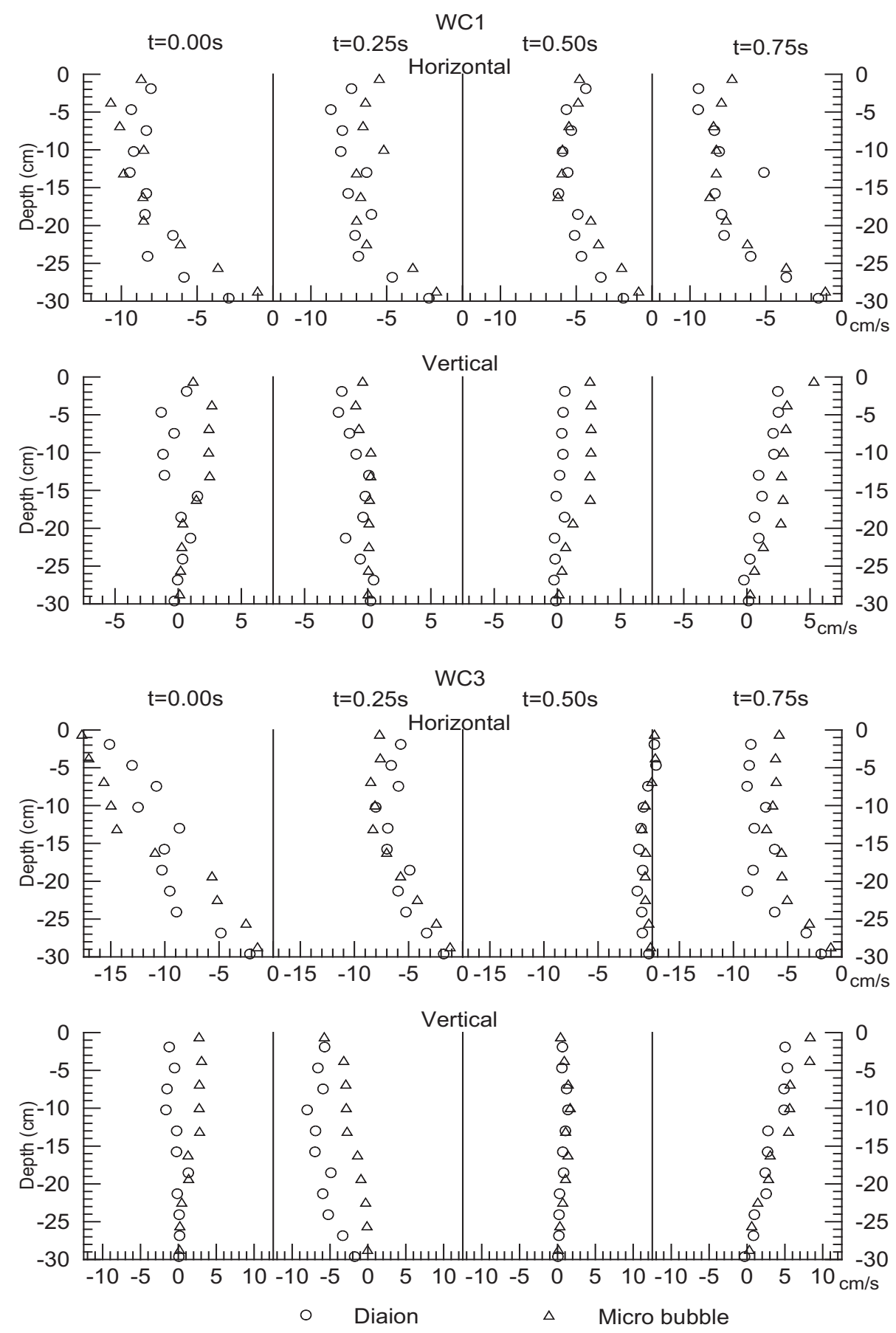

Figure 6 Horizontal and vertical velocity profiles for wave-current cases. 
Figure 7 displays a plot of particle orbit geometries at several depths in the wave-alone cases. The circular symbol indicates the instantaneous position of a water particle based on the present algorithm (see Figure 2) in the PIV measurement with DIAION. The solid curve shows the theoretical trajectory by Eqs. 25 and 26 whose higher terms lead to an asymmetry of particle orbit. This asymmetry increases with an increase in the wave height. Inspecting these comparisons, the experimental data generally agree with the theoretical trajectory in the total depth except the near-surface region for Run W2. Closer to the bottom, the trajectory is rather thinner and flatter due to the larger horizontal excursion and smaller vertical excursion. The experimental orbital motion diminishes more quickly than the theoretical one, and this tendency is prominent for the larger wave height. The trajectory seems to be closed, but the particle at an intermediate elevation moves slightly backward from the wave direction to maintain a balance between the following and opposing fluxes in the wave tank. Note that ideal trajectory data cannot be obtained without removing extraneous effects: i.e. reflection from the wall, higher harmonics generated by the wavemaker and etc. Figure 8 shows the water particle trajectories for waves with a steady current. As the current speed is faster than the water particle velocity due to the wave-alone motion, the particles travel further. The particle trajectory takes a Stokes-wave type form for Run WC1, a nearly cusped profile for Run WC2, and a looped path for Run WC3. The stagnant section of the trajectory occurs when the wave trough arrives, but the water particle advances in most part of a wave cycle. We note that a small change in the wave height is sufficient to dramatically modify the type of the trajectory. As particles near the bottom take less distance to complete one wave cycle when compared to those near the water surface, smaller value of current velocity is given for the nearbottom particles to calculate the horizontal and vertical displacements with Eqs. 25 and 26.

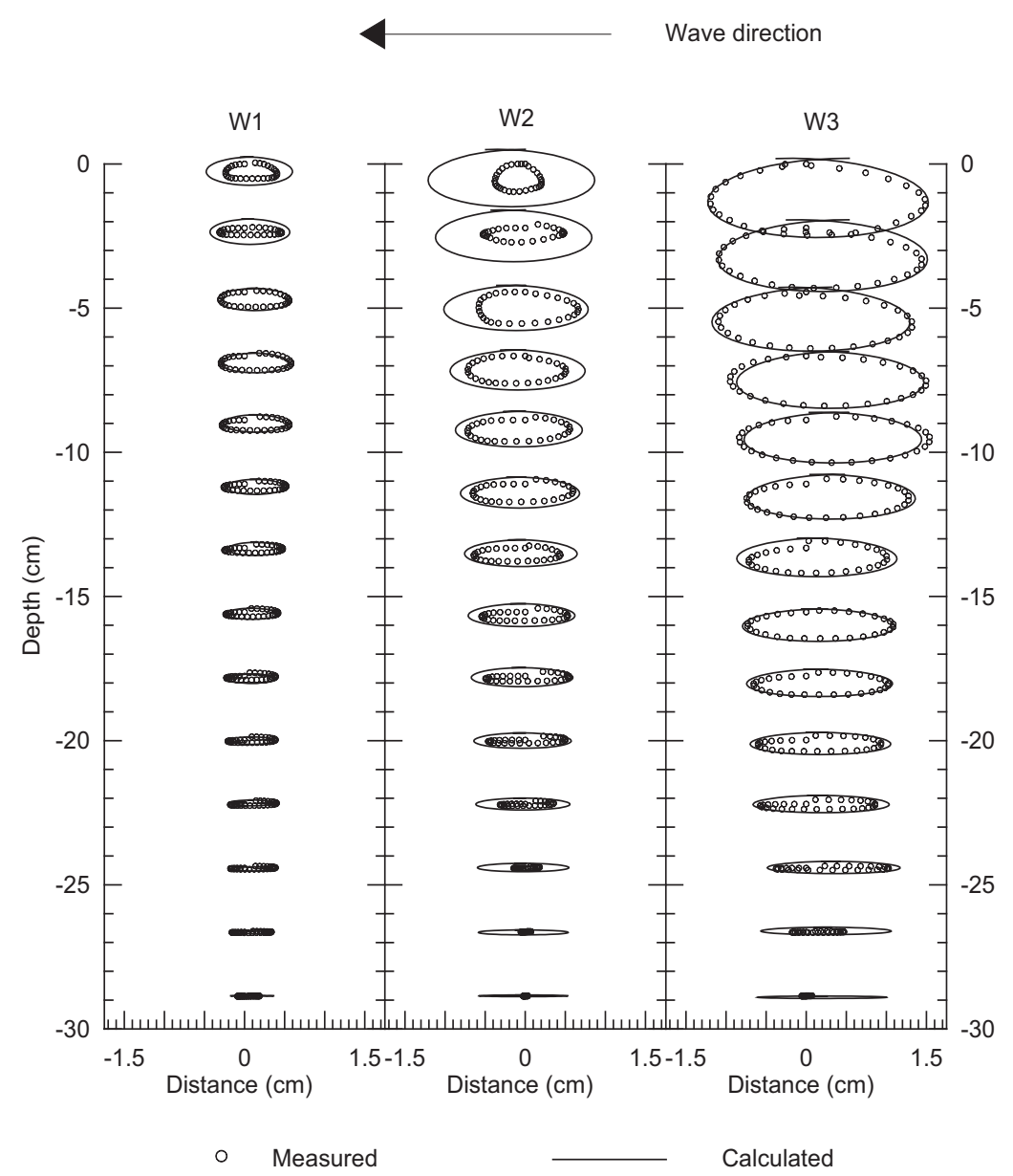

Figure 7 Comparisons between theoretical and experimental water particle trajectoies for wave-alone cases. 


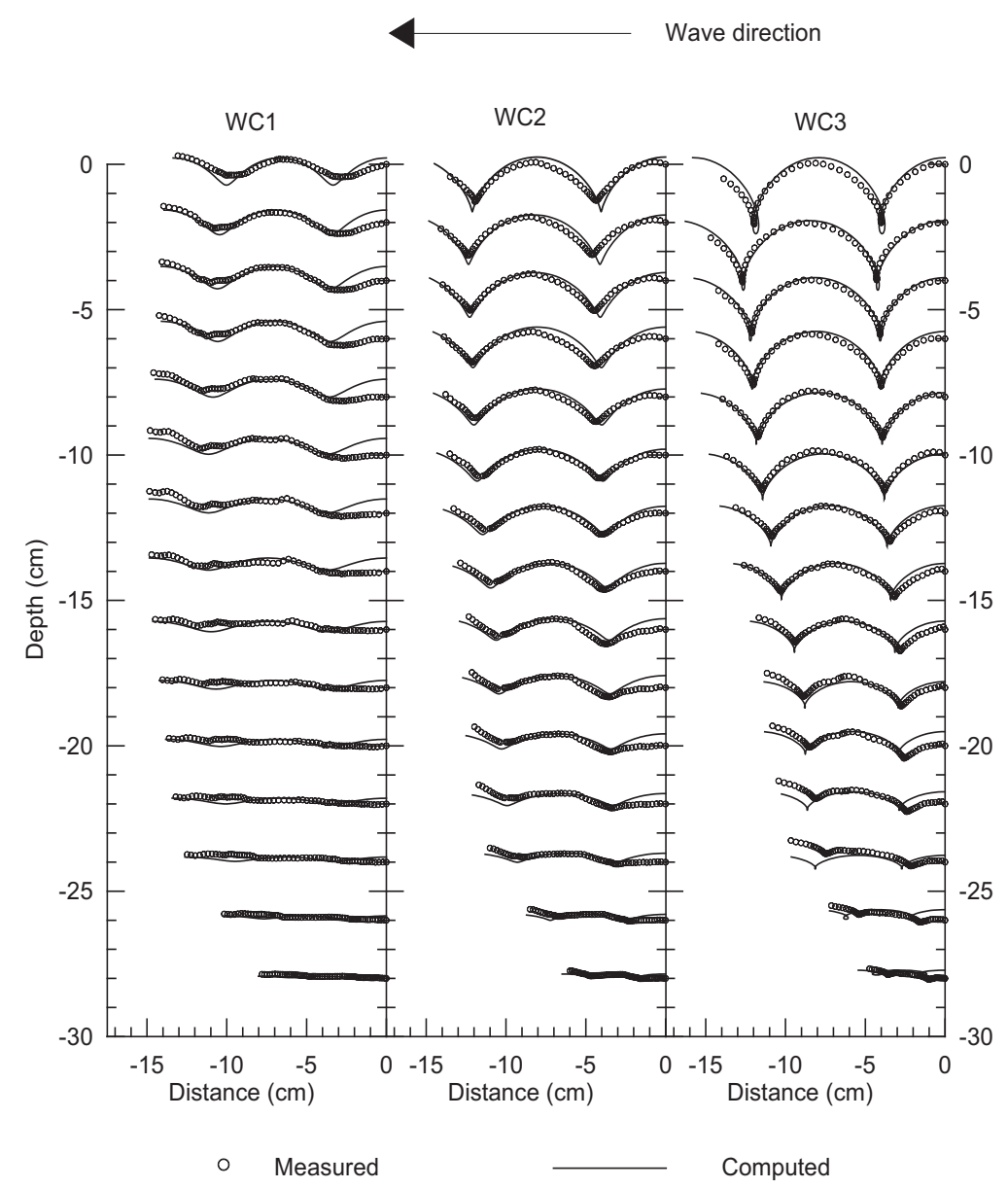

Figure 8 Comparisons between theoretical and experimental water particle trajectoies for wave-current cases.

\section{CONCLUSIONS}

We theoretically and experimentally investigated the velocity distributions for Stokes waves propagating with and without a steady current. The theory is consisted of the third-order perturbation solution for computing the flow of Stokes waves in the Eulerian scheme. The particle trajectories of a flow were simulated using a solution based on the definition of Lagrangian approach to the second order of a Taylor series expansion. The water particle velocity was observed using a PIV system, in which we tried out two different tracers such as DIAION and micro bubbles, and successive velocity fields over one wave cycle were obtained for some wave heights. The instantaneous velocity vector map clearly illustrated a pair of vortices in the wave-alone condition. The EC meter was then used to measure the horizontal and vertical velocity components at a cross section, and these vertical distributions at an arbitrary phase were compared with the PIV data given through DIAION as well as the results computed by the Stokes third-order theory. A suitable agreement between these experimental and theoretical results confirms the accuracy of the PIV measuring technique. Validation results were also presented in a wave-current motion from the comparison between DIAION and micro bubbles. While the PIV measurements in the different tracers were quite good, they were clearly affected by the fluctuations associated with the gravity and buoyancy. Accurate evaluation of the mass-transport of Stokes waves is significant in determining the Lagrangian residual velocity, in addition to the basic use of PIV technique. We demonstrated that the algorithm for PIV be employed to compute Lagrangian velocity and to track water particle displacements in Eulerian grids. Good agreement between the computed and measured results shows the high accuracy of the proposed approach that can be applied to the Lagrangian description of the trajectory of a water particle. 


\section{REFERENCES}

Adrian, R.J. 1991. Particle imaging techniques for experimental fluid mechanics, Annual Review of Fluid Mech., 23, 261-304.

Airy, G.B. 1845. On tides and waves, Encyclopedia Metro., London, 241-396.

Borgman, L.E., and J.E. Chappelear. 1958. The use of the Stokes-Struik approximation for waves of finite height, Proceedings of $6^{\text {th }}$ International Conference on Coastal Engineering, ASCE, 252-280.

Constantin, A. 2006. The trajectories of particles in Stokes waves, Inventions Mathematicae, 166, 523535.

Constantin, A., \& W. Strauss. 2010. Pressure Beneath a Stokes Wave, Communications on Pure and Applied Mathematics, 63, 533-557.

Fenton, J.D. 1985. A fifth-order Stokes theory for steady waves, Journal of Waterway, Port, Coastal and Ocean Engineering, ASCE, 111(2), 216-234.

Grue, J., P. L-F. Liu \& G. K. Pedersen. 2004. PIV and Water Waves, Advances in Coastal and Ocean Engineering, World Scientific, New Jersey, 9, 339pp, 531 pp.

Shimizu, R., Shintani, T. and M. Umeyama. 2006. Instantaneous and Lagragian velocity fields of internal waves on a slope by PIV measurement and numerical simulation, Annual Journal of Coastal Engineering, JSCE, 52, 1-5.

Skjelbreia, L., and J. Hendrickson. 1961. Fifth order gravity wave theory, Proceedings of $7^{\text {th }}$ International Conference on Coastal Engineering, ASCE, 184-196.

Stokes, G.G. 1847. On the theory of oscillatory waves, Trans. Cambridge Phil. Soc., 8 (441).

Umeyama, M. 2005. Reynolds stresses and velocity distributions in a wave-current coexisting environment, Journal of Waterway, Port, Coastal and Ocean Engineering, ASCE, 131(5), 203212.

Umeyama, M. 2008. PIV techniques for velocity fields of internal waves over a slowly varying bottom topography, Journal of Waterway, Port, Coastal and Ocean Engineering, ASCE, 134(5), 286-298.

Umeyama, M. 2009a. Mean velocity changes due to interaction between bichromatic waves and a current, Journal of Waterway, Port, Coastal and Ocean Engineering, ASCE, 135(1), 11-23.

Umeyama, M. 2009b. Changes in turbulent flow structure under combined wave-current motions, Journal of Waterway, Port, Coastal and Ocean Engineering, ASCE, 135(5), 213-227.

Umeyama, M. 2010. Coupled PIV and PTV measurements of particle velocities and trajectories for surface waves following a steady current, Journal of Waterway, Port, Coastal and Ocean Engineering, ASCE, in press.

Umeyama, M., and H. Shinomiya. 2009. Particle image velocimetry measurements for Stokes progressive internal waves, Geophysical Research Letters, 36(6), AGU, L06603.

Wallet, A. and F. Ruellan. 1950. Trajectoires internes dans un clapotis partiel, La Houille Blanche, 5, 483-489.

Willert, C.E. and M. Gharib. 1991. Digital particle image velocimetry, Experiments in Fluids, 10, 181193. 